\title{
Effects of sugammadex plus rocuronium vs neostigmine plus cisatracurium during renal transplantation on graft function: a retrospective, case-control study
}

Maria Vargas ( $\nabla$ vargas.maria82@gmail.com )

Universita degli Studi di Napoli Federico II https://orcid.org/0000-0001-7652-970X

\section{Pasquale Buonanno}

Universita degli Studi di Napoli Federico II Dipartimento di Neuroscienze e Scienze Riproduttive ed Odontostomatologiche

\section{Andrea Sica}

Universita degli Studi di Napoli Federico II Dipartimento di Neuroscienze e Scienze Riproduttive ed Odontostomatologiche

\section{Emanuele Sabatella}

Universita degli Studi di Napoli Federico II Dipartimento di Neuroscienze e Scienze Riproduttive ed Odontostomatologiche

\section{Francesco P. D’Alessio}

Universita degli Studi di Napoli Federico II Dipartimento di Neuroscienze e Scienze Riproduttive ed Odontostomatologiche

\section{Simone Alfieri}

Universita degli Studi di Napoli Federico II Dipartimento di Neuroscienze e Scienze Riproduttive ed Odontostomatologiche

\section{Carmine lacovazzo}

Universita degli Studi di Napoli Federico II Dipartimento di Neuroscienze e Scienze Riproduttive ed Odontostomatologiche

\section{Rosa Carrano}

Universita degli Studi di Napoli Federico II

\section{Giuseppe Servillo}

Universita degli Studi di Napoli Federico II Dipartimento di Neuroscienze e Scienze Riproduttive ed Odontostomatologiche

\section{Research article}

Keywords: Renal function, neuromuscular blockade agent, renal transplantation, graft function 
Posted Date: November 4th, 2019

DOI: https://doi.org/10.21203/rs.2.14392/v2

License: (c) (i) This work is licensed under a Creative Commons Attribution 4.0 International License. Read Full License

Version of Record: A version of this preprint was published at Transplantation Proceedings on April 1st, 2021. See the published version at https://doi.org/10.1016/j.transproceed.2020.09.012. 


\section{Abstract}

Background: Rocuronium can be used in patients with severe renal failure (creatinine clearance $<30 \mathrm{ml} / \mathrm{min}$ ), but the duration of muscle relaxation is longer and results in an increased risk of postoperative residual curarization. Rocuronium can be antagonized by sugammadex, but the elimination of the complex they make (rocuronium-sugammadex complex) varies according to the renal function. Two case reports/series have reported the use of rocuronium-sugammadex complex during renal transplantation. This retrospective case-control study aims to investigate the effects of rocuroniumsugammadex, used during renal transplantation, on transplanted kidney function.

Methods: We analyzed 113 medical records of patients undergoing kidney transplantation from January 2015 to December 2018. Forty-seven medical records were excluded because they did not report the administration of rocuronium + sugammadex (roc + sug) or cisatracurium + neostigmine (cis + neo) during the transplantation. The demographics of patients and donors were collected along with the following data: blood urea and creatinine, serum and urinary electrolytes, and diuresis. Marginal, single or double kidney transplantations, Karpinsky score and histologic evaluation of transplanted kidney were collected.

Results: We finally collected data from 66 medical reports from January 2015 to December 2018. Blood creatinine levels at $6 \mathrm{hrs}, 12 \mathrm{hrs}$ and $24 \mathrm{hrs}$ were significantly lower in the roc + sug group than in the cis + neo group (crea 6 hrs $=0.05$, crea 12 hrs $p=0.038$, crea 24 hrs $p=0.049$ ). Blood urea levels for 24 hrs after transplantation were significantly lower in the roc + sug group than in the cis + neo group (urea 0 hrs $p=0.025$, urea 6 hrs $p=0.011$, urea 12 hrs $p=0.03$, urea 24 hrs $p=0.011$ ). We found no statistically significant differences in blood sodium, blood potassium, blood calcium, diuresis, urinary sodium, or urinary potassium levels before and after transplantation.

Conclusions: In this retrospective case-control study, the use of rocuronium and sugammadex during renal transplant surgery did not affect the recovery of the graft function during the first week after transplantation.

\section{Introduction}

Rocuronium may be used in patients with severe renal failure (creatinine clearance $<30 \mathrm{ml} / \mathrm{min}$ ), but the duration of muscle relaxation is longer and results in an increased risk of postoperative residual curarization $[1,2]$. Rocuronium can be antagonized by sugammadex, but the elimination of the complex they make (rocuronium-sugammadex complex) varies according to the renal function [3].

In patients with end-stage renal failure, sugammadex $4 \mathrm{mg} / \mathrm{kg}$ is able to clinically reverse deep neuromuscular blockade and it is as rapid as in healthy controls [4]. In a recent study Panhuizen et al. reported that sugammadex $4 \mathrm{mg} / \mathrm{kg}$ resulted in a complete reversal of deep rocuronium-induced neuromuscular blockade in patients with normal and severely impaired renal function, even if the time from start of sugammadex to recovery of the $\mathrm{T} 4 / \mathrm{T} 1$ ratio to 0.9 was slower in the renal group [5]. 
Different studies have demonstrated that the pharmacokinetics of sugammadex was significantly altered by chronic renal failure, since the kidney is the major excretory organ of this drug [4]. In a phase III study evaluating the PK of the rocuronium-sugammadex complex, plasma concentrations of sugammadex dropped slower in the renal failure group compared to the control group [6]. Staals et al. have reported that "total plasma clearance of sugammadex was 17 times lower and the mean elimination half-life was 16 times higher in the renal failure group" [6]. In the case of renal impairment, the rocuroniumsugammadex complex may be retained for a long time after its complete elimination [6]. The rocuroniumsugammadex complex still indicated a measurable concentration seven days after its administration [5]. Considering the prolonged half-life of sugammadex in patients with severe renal impairment, it is not safe to recommend its use in patients with renal failure [5].

Patients with end stage renal failure are candidates for renal transplantation. During renal transplant surgery, anesthesiologists should avoid using nephrotoxic drugs [7]. During renal transplantation, muscle relaxation can otherwise be achieved with different drugs eliminated in the presence of renal failure [7]. According to this, atracurium and cisatracurium, undergoing Hofmann degradation and ester hydrolysis, may be used. Although rocuronium may be reversed with sugammadex at the end of surgery, excretion of the rocuronium-sugammadex complex is kidney dependent [7]. The product data sheet does not recommend the use of rocuronium in patients with renal failure until future research has evaluated its safety and tolerability profile [7]. Two case reports/series without control groups reported the use of the rocuronium-sugammadex complex during renal transplantation, but they were mainly focused on the evaluation of rocuronium-sugammadex activities $[8,9]$. Our case-control study aimed to compare the effect on renal function of rocuronium-sugammadex versus cisatracurium-neostigmine after kidney transplantation in order to determine the best neuromuscular block management in this scenario.

\section{Methods}

The study was approved by the IRB of Federico II University of Naples, Naples, Italy (protocol number 113/19), and was conducted in compliance with the Declaration of Helsinki (1964 and following amendments), current Good Clinical Practices, and the applicable European and local regulatory requirements.

Patients in our university hospital are usually invited to freely give written informed consent to authorize the use of their clinical data for research purposes. All subjects included in this study provided consent for the use of their data and the local IRB waived the requirement for further written informed consent.

\section{Subjects}

This was a retrospective, case-control study conducted at Federico II University Hospital of male and female patients undergoing general anesthesia for kidney transplantation. Inclusion criteria were age $\geq$ 18 , years, use of sugammadex + rocuronium or neostigmine + cisatracurium during the transplantation, collection of blood, renal and urinary parameters for the first week after the transplantation, and no 
transplant rejection and/or removal of transplanted kidney during the first week. Exclusion criteria were age $<18$ years, transplant rejection or removal of transplanted kidney during the first week and concomitant use of nephrotoxic drugs.

We analyzed 113 medical records of patients undergoing kidney transplantation from January 2015 to December 2018. Forty-seven medical records were excluded, because they did not report the administration of rocuronium + sugammadex (roc + sug) or cisatracurium + neostigmine $(c i s+$ neo $)$ during the transplantation. All patients had received the graft from a cadaveric donor. We finally collected data from 66 medical reports.

\section{Patient management}

Anesthesia was induced with 1.5 to $2.5 \mathrm{mg} / \mathrm{kg}$ propofol and 1 to $2 \mu \mathrm{g} / \mathrm{kg}$ fentanyl. After acceleromyograph calibration, a single dose of $0.6 \mathrm{mg} / \mathrm{kg}$ rocuronium or $0.2 \mathrm{mg} / \mathrm{kg}$ cisatracurium was administered and tracheal intubation was performed. Additional doses of $0.15 \mathrm{mg} / \mathrm{kg}$ rocuronium or 0.03 $\mathrm{mg} / \mathrm{kg}$ cisatracurium were administered during surgery at TOF2 if required. Anesthesia was maintained by sevoflurane and remifentanil and titrated according to hemodynamic and autonomic responses. At the end of surgery, sevoflurane was decreased to an end-tidal concentration of $0.8 \%$ to $1 \%$, and $2 \mathrm{mg} / \mathrm{kg}$ sugammadex or $0.05 \mathrm{mg} / \mathrm{kg}$ neostigmine was administered when the acceleromyograph registered a TOF2 response. Methylprednisolone was given intravenously at a dosage of $1 \mathrm{gr}$ at the unclamping of the arterial vessels, $150 \mathrm{mg}$ on the first day after the transplantation, $75 \mathrm{mg}$ on the second day, $35 \mathrm{mg}$ on the third day, and $16 \mathrm{mg}$ on the fourth day. Furosemide was administered at a dosage of 3 to $5 \mathrm{mg} / \mathrm{kg}$ when needed.

\section{Data collection and outcome}

For each medical record the following data were recorded: age, gender, reason for chronic renal failure, body mass index (BMI), comorbidities, type and dose of neuromuscular blockers and proper reversals, donor age, ischemia time, need for pre- and post-operative dialysis, and post-operative bleeding. Blood creatinine, blood urea, blood sodium, blood potassium, and blood calcium levels were collected on the day before the surgery, the day of the surgery, after 6 hours (hrs), 12 hrs, 24 hrs, 48 hrs and 72 hrs.

Diuresis, urinary sodium and urinary potassium levels were collected on the day of the surgery, after $6 \mathrm{hrs}$, $12 \mathrm{hrs}, 24 \mathrm{hrs}, 48 \mathrm{hrs}$ and $72 \mathrm{hrs}$. Marginal, single or double kidney transplantations, Karpinsky score and histologic evaluation of the transplanted kidney were collected.

The primary outcome of this study was to evaluate the impact of using rocuronium + sugammadex vs cisatracurium + neostigmine during kidney transplantation on the recovery of kidney function evaluated in terms of serum creatinine and serum urea variations. The secondary outcome was to evaluate whether the use of rocuronium + sugammadex or cisatracurium + neostigmine were risk factors for kidney injury 
after kidney transplantation. Acute kidney injury after kidney transplantation was evaluated according KDIGO criteria as increase of creatinine levels to $0.3 \mathrm{mg} / \mathrm{dl}$ after 48 hours.

\section{Statistical analysis}

Data are presented as means \pm SDs or median and range interquartile. Data were analyzed with one-way analysis of variance, and distribution of residuals was investigated using the Shapiro-Wilk test. Comparison between groups was performed with ANOVA for repeated measurement. Any comparison was weighted according the sample size. The Cohen's d effect size was further calculated on the comparison that reached a statistical significance. The effect size was classified as small $(<0.2)$, medium (0.2-0.8) and large (>0.8). A univariate logistic regression was performed to identify clinically meaningful variables potentially associated with the risk of post-transplantation renal failure. Thereafter, multiple logistic regressions were carried out using backward stepwise variable elimination, including the variables with a $p<0.05$ at the univariate analysis. Statistical significance was set at $p=0.05$. Statistical analysis was performed using SPSS (version 20.0, IBM®, USA).

\section{Results}

Forty-seven medical records were excluded because they did not report the administration of roc + sug or cis + neo during the transplantation. We finally collected data from 66 medical reports from January 2015 to December 2018. Thirty patients had been treated with roc + sug and 36 patients with cis + neo. Table 1 presents the baseline characteristics of enrolled patients. There were no differences in age, gender, BMI, ischemic time, donor's age, creatinine levels and uremic levels on the day before the transplantation, the need for postoperative dialysis and Karpinsky score.

Figure 1 illustrates the blood creatinine levels before and after the kidney transplantation. Blood creatinine levels at $6 \mathrm{hrs}, 12 \mathrm{hrs}$ and $24 \mathrm{hrs}$ were significantly lower in the roc + sug group than in the cis + neo group (crea 6 hrs $p=0.05$, crea 12 hrs $p=0.038$, crea 24 hrs $p=0.049$ ). Figure 2 illustrates the blood urea levels before and after the transplantation. Blood urea levels for $24 \mathrm{hrs}$ after the transplantation were significantly lower in the roc + sug group than in the cis + neo group (urea 0 hrs $p=0.025$, urea 6 hrs $p=$ 0.011 , urea $12 \mathrm{hrs} p=0.03$, urea $24 \mathrm{hrs} p=0.011$ ). Medium effect sizes were found for the comparisons between groups of the creatinine levels at $6 \mathrm{hrs}, 12 \mathrm{hrs}$ and $24 \mathrm{hrs}$ (Cohen's d crea $6 \mathrm{hrs}=0.51$, Cohen's $d$ crea $12 \mathrm{hrs}=0.53$, Cohen's d crea $24 \mathrm{hrs}=0.50$ ) and of urea levels from 0 to $24 \mathrm{hrs}$ (Cohen's d urea $0 \mathrm{hrs}$ $=0.58$, Cohen's d urea $6 \mathrm{hrs}=0.67$, Cohen's d urea $12 \mathrm{hrs}=0.54$, Cohen's d urea $24 \mathrm{hrs}=0.68$ ). We found no statistically significant differences in blood sodium, blood potassium, blood calcium, diuresis, urinary sodium, or urinary potassium levels before and after the transplantation (Figure 3 and Figure 4).

According to KDIGO criteria, an increase in creatinine levels of $0.3 \mathrm{mg} / \mathrm{dl}$ after 48 hours was found in 27 patients of th cis + neo group and in 16 patients of the roc + sug group. Table 2 presents the univariate and multivariate regression. The use of roc + sug or cis + neo was not an independent risk factor for acute kidney injury after the transplantation. In the multivariate analysis, we found that the risk of acute 
kidney injury after the transplantation was: 1 ) independently associated with the blood urea level at 48 hrs-this means that a blood urea level at $48 \mathrm{hrs}$ of $142 \mathrm{mg} / \mathrm{dl}$ (median, IQ range 113-187) increased the risk of having an acute kidney injury by $5 \%$; and 2 ) the blood creatinine level at $24 \mathrm{hrs}$ was a protective factor-this means that a blood creatinine level at 24 hrs of 3.40 (median, IQ range 2.6-4.8) decreased the risk of having an acute kidney injury by $11,7 \%$.

\section{Discussion}

To the best of our knowledge this is the first study evaluating the influence of rocuronium and sugammadex, compared to cisatracurium and neostigmine, on graft function after renal transplantation. We retrospectively analyzed medical records of patients who had undergone kidney transplantation, whose neuromuscular block was reached by cisatracurium or rocuronium and the reversal obtained by neostigmine and sugammadex, respectively. We found that: 1) during the first 24 hours after the transplantation, blood creatinine and urea levels were significantly lower in the rocuronium-sugammadex group; 2) there was no difference in serum and urinary electrolyte levels between the considered groups; and 3 ) the use of both neuromuscular agents and their reversals were not independent risk factors for acute kidney injury after the transplantation.

Creatinine is commonly used as a measure of kidney function [10]. The diagnosis of renal failure is usually suspected when serum creatinine in greater than the upper limit of the normal range [10]. In the early post-transplant period the graft function also depended on the donor characteristics [10]. In this study, variables that may affect the graft function were the donor's age, the ischemic time and the Karpinski score, which were not different between the groups and, interestingly, the graft function was well restored during the first 72 hours after the transplantation, since the blood creatinine levels decreased as expected [10]. However, this happened much more often in the sugammadex group, even if it was rapidly cleared unchanged via glomerular filtration without tubular secretion, absorption or metabolism [11].

During the renal transplantation surgery, the unclamping of the arterial vessels resulted in reperfusion of the transplanted kidney with an immediate urine production in more than $90 \%$ of patients. This is proof of the restoration of glomerular function. Theoretically, once the glomerular filtration was resumed in the early post-operative period, sugammadex may be easily excreted by the transplanted kidney [12]. In our patients, the serum creatinine levels and diuresis were fully restored suddenly after the first 24 hours following the transplantation, even in patients treated with rocuronium and sugammadex. This may be due to the fact that the rocuronium-sugammadex complex may be removed according their physiologic pharmacokinetics after the restoration glomerular filtration. Accordingly, there was no increase in serum creatinine levels in the rocuronium-sugammadex group. Indeed, the serum creatinine levels were lower in the rocuronium group compared to the cisatracurium group, even if the cisatracurium underwent Hofmann degradation and ester hydrolysis. 
In this study, blood urea levels were significantly lower in the rocuronium-sugammadex group, supporting the good recovery of the graft function. However, we found a slight increase in blood urea levels during the first three days after transplantation. This finding may have many explanations. The transplanted patients received corticosteroids to prevent graft rejection, which has a catabolic action which may contribute to serum urea rising $[13,14]$. Furthermore, to counteract acute tubular necrosis (ATN), kidney transplanted patients were treated with diuretic drugs that may increase urea levels $[15,16,17]$.

Serum and urinary electrolytes did not indicate any significant difference between the two groups. The modification of their concentrations was in accordance with the restored kidney function. Potassium excretion was demonstrated by its increase in urinary levels and the concurrent decrease of its blood concentrations. Ghoneim et al. have reported similar sodium and potassium serum concentrations in pediatric patients undergoing general anesthesia and treated with sugammadex or neostigmine [18].

In this study we found that the use of both neuromuscular agents and their reversals were not independent risk factors for acute kidney injury after transplantation, while proper renal biomarkers, such as blood creatinine and urea levels, may play a role $[19,20]$. Isik et al. have reported an increase of the renal biomarker cystatin $\mathrm{C}$ in patients undergoing general anesthesia and treated with neostigmine, but not in patients receiving sugammadex for neuromuscular block reversal. The authors concluded that sugammadex indicated a higher tolerability than neostigmine. The authors also affirmed that neither drug causes renal failure but only a subclinical kidney damage; in fact, the effect on kidney function was evidenced only by cystatin $\mathrm{C}$ but no clinical signs of renal failure or changes in creatinine, blood urea nitrogen, sodium, potassium, or calcium were recorded, since in both groups they found a significant reduction in post-operative serum calcium concentration, as we also did in our study [21]. The better renal function exhibited by the roc+sug group compared to the cis+neo group is also supported by in vitro studies, which indicate that neostigmine is more cytotoxic and genotoxic than sugammadex, causing apoptosis and necrosis of human embryonic renal cells [22]. Sugammadex, by comparison, indicated a protective effect against ischemia-reperfusion injury in an in vivo model of cerebral ischemia, probably due to the gamma cyclodextrin ring of sugammadex [23]. This beneficial effect can partly explain the better renal function after transplantation observed in our study in the group receiving sugammadex, even if the exact mechanism still has to be elucidated. Detrimental effects of sugammadex on renal function have been reported in the literature. Histological changes were found and alteration of more sensitive kidney function markers (i.e. N-acetyl-beta-glucosaminidase, microalbuminuria, and beta 2-microglobulin) were observed, but in all cases there was no evidence of clinically evident damage $[24,25,26]$. Other studies did not confirm the alterations of both classical and new renal damage biomarkers associated with the use of sugammadex and neostigmine [27].

In this study we found that a blood urea level at 48 hours of $142 \mathrm{mg} / \mathrm{dl}$ (median, IQ range 113-187) increased the risk of having an acute kidney injury by $5 \%$, while a blood creatinine level at 24 hours of 3.40 (median, IQ range $2.6-4.8$ ) decreased the risk of having an acute kidney injury by $11.7 \%$. To find general risk factors for acute kidney injury after kidney transplantation was beyond the aim of this study. We performed a multivariate analysis with the aim to assess whether the rocuronium plus sugammadex 
might be a risk factor for kidney injury after renal transplantation. The role of different creatinine levels after renal transplantation has been discussed in various studies that have found a general agreement between the levels of serum creatinine and graft failure [28]. In general, a longitudinal increase of serum creatinine levels may be used to predict graft failure [29]. A recent study has found that one unit increase in serum creatinine level suggests a fourfold increased risk of graft failure [29]. Our data support the close relationship between serum creatinine and graft function.

Finally, we found no difference in the need for post-operative dialysis between the considered groups. The need for post-operative dialysis may not be worsened by the use of rocuronium + sugammadex or cisatracurium + neostigmine.

This study has many limitations. We did not measure hemodynamic changes during surgery and in the post-operative period. The graft perfusion can be an important factor affecting transplanted kidney function. However, no significant differences in hemodynamic parameters between neostigmine and sugammadex have been reported in previous studies [30,31,32].

Furthermore, since our analysis was conducted retrospectively, we investigated only routine biomarkers of renal function; probably more detailed information about graft damage may be drawn from more sensitive biomarkers, such as NAG, urinary albumin, and beta2-microglobulin.

\section{Conclusions}

In this retrospective case-control study, the use of rocuronium and sugammadex during renal transplant surgery did not affect the recovery of the graft function in terms of serum creatinine and blood urea increase during the first week after the transplantation. However, prospective and randomized studies are needed to further confirm this finding.

\section{Abbreviations}

rocuronium + sugammadex $=$ roc + sug

cisatracurium + neostigmine $=$ cis + neo

hours $=$ hrs

body mass index $=\mathrm{BMI}$

\section{Declarations}

\section{Ethics approval and consent to participate}

The study was approved by the IRB of Federico II University of Naples, Naples, Italy (protocol number 113/19). All subjects included in this study provided consent for the use of their data and the local IRB 
waived the requirement for further written informed consent.

\section{Consent for publication}

N/A

\section{Availability of data and materials}

The datasets used and/or analyzed during the current study are available from the corresponding author on reasonable request.

\section{Competing interest}

The authors have no financial competing interest from this study.

\section{Funding}

The authors received no funding for this study.

\section{Authors' contributions}

$\mathrm{MV}, \mathrm{PB}, \mathrm{AS}, \mathrm{Cl}, \mathrm{RC}$ and GS helped to write the protocol for the study, to collect the data, to analyze the data, to write the manuscript and to approve the final version. ES, FDP and SA helped to collect the data, to analyze the data, to write the manuscript and to approve the final version.

\section{Acknowledgements}

$\mathrm{N} / \mathrm{A}$

\section{References}

- Robertson EN, Driessen JJ, Booij LH: Pharmacokinetics and pharmacodynamics of rocuronium in patients with and without renal failure. Eur $J$ Anaesthesiol 2005; 22:4-10.

- Murphy GS, Szokol JW, Marymont JH, et al.: Residual neuromuscular blockade and critical respiratory events in the postanesthesia care unit. Anesth Analg 2008; 107:130-137.

- Update on the intraoperative management of adult cadaveric renal transplantation; BJA Education 2016;16:53-57

- de Souza CM, Tardelli MA, Tedesco H, et al: Efficacy and safety of sugammadex in the reversal of deep neuromuscular blockade induced by rocuronium in patients with end-stage renal disease: $\mathrm{A}$ 
comparative prospective clinical trial. Eur J Anaesthesiol. 2015; 10:681-6.

- Panhuizen IF, Gold JA, Buerkle C et al. Efficacy, safety and pharmacokinetics of sugammadex $4 \mathrm{mg}$ kg-1 for reversal of deep neuromuscular blockade in patients with severe renal impairment. Br J Anaesth 2015; 114:777-84

- Staals LM, Snoeck MMJ, Driessen JJ et al. Reduced clearance of rocuronium and sugammadex in patients with severe to end-stage renal failure: a pharmacokinetic study. Br J Anaesth 2010; 104:319

- Schmid S1, Jungwirth B: Anaesthesia for renal transplant surgery: an update. Eur J Anaesthesiol 2012; 29:552-558.

- Carlos RV, Torres ML, de Boer HD: The use of rocuronium and sugammadex in paediatric renal transplantation: two case reports. Eur J Anaesthesiol 2016; 33:383-385.

- Ono A, Fujita Y, Kajiura T, et al.: Efficacy and safety of sugammadex in patients undergoing renal transplantation. JA Clinical Reports 2018; 4:56.

- Govani MV1, Kwon O, Batiuk TD, Milgrom ML, Filo RS: Creatinine reduction ratio and 24-hour creatinine excretion on post transplant day two: simple and objective tools to define graft function. $J$ Am Soc Nephrol. 2002; 6:1645-9.

- Cammu G, Van Vlem B, van den Heuvel M, et al.: Dialysability of sugammadex and its complex with rocuronium in intensive care patients with severe renal impairment. Br J Anaesth 2012; 109:382390.

- Peeters P, Passier P, Smeets J, et al.: Sugammadex is cleared rapidly and primarily unchanged via renal excretion. Biopharm Drug Dispos 2011; 32:159-167.

- Wolthers T, Hamberg O, Grøfte T, Vilstrup H.: Effects of budesonide and prednisolone on hepatic kinetics for urea synthesis. J Hepatol. 2000; 4:549-54.

- Wolthers T, Grøfte T, Jørgensen JO, Vilstrup H.: Growth hormone prevents prednisolone-induced increase in functional hepatic nitrogen clearance in normal man. J Hepatol. 1997; 5:789-95.

- McCabe R, Stevens LE, Subramamian A, Lattes C, Hashim GM.: Reduction of acute tubular necrosis (ATN) by furosemide and steroids in cadaveric kidney recovery. Am J Surg. 1975; 3:246-8.

- Nierenberg DW.: Furosemide and ethacrynic acid in acute tubular necrosis. West J Med. 1980; 2:163-70.

- Peterson OW, Gabbai FB, Myers RR, Mizisin AP, Blantz RC.: A single nephron model of acute tubular injury: role of tubuloglomerular feedback. Kidney Int. 1989; 6:1037-44.

- Ghoneim AA, El Beltagy MA: Comparative study between sugammadex and neostigmine in neurosurgical anesthesia in pediatric patients. Saudi J Anaesth, 2015; 9: 247-52.

- Lemmens HJ, El-Orbany MI, Berry J et al.: Reversal of profound vecuronium-induced neuromuscular block under sevoflurane anesthesia: Sugammadex versus neostigmine. BMC Anesthesiol, 2010; 10: 5. 
- Rahe-Meyer N, Fennema H, Schulman S et al.: Effect of reversal of neuromuscular blockade with sugammadex versus usual care on bleeding risk in a randomized study of surgical patients. Anesthesiology, 2014; 121: 969-77.

- Isik Y, Palabiyik O, Cegin BM, Goktas U, Kati I: Effects of Sugammadex and Neostigmine on Renal Biomarkers. Med Sci Monit. 2016 Mar 10; 22:803-9.

- Büyükfırat E, Koyuncu I, Karahan MA et al. Comparison of the cytotoxic, genotoxic and apoptotic effects of Sugammadex and Neostigmine on human embryonic renal cell (HEK-293). Cell Mol Biol 2018;64(13):74-78.

- Ozbilgin S, Yılmaz O, Ergur BU, et al. Effectiveness of sugammadex for cerebral ischemia/reperfusion injury. Kaohsiung J Med Sci. 2016;32:292-30

- Bostan H, Kalkan Y, Tomak Y et al: Reversal of rocuronium-induced neuromuscular block with sugammadex and resulting histopathological effects in rat kidneys. Renal Failure, 2011;33: 101924.

- Sparr HJ, Vermeyen KM, Beaufort AM et al.: Early reversal of profound rocuronium-induced neuromuscular blockade by sugammadex in a randomized multicenter study efficacy, safety and pharmacokinetics. Anesthesiology, 2007; 106: 935-43.

- Sorgenfrei IF, Norrild K, Larsen PB et al.: Reversal of rocuronium-induced neuromuscular block by the selective relaxant binding agent sugammadex: A dose-finding and safety study. Anesthesiology, 2006; 104: 667-74.

- Khuenl-Brady KS, Wattwil M, Vanacker BF et al.: Sugammadex provides faster reversal of vecuronium-induced neuromuscular blockade compared with neostigmine: A multicenter, randomized, controlled trial. Anesth Analg, 2010; 110: 64-73.

- Debout A, Foucher Y, Trebern-Launay K et al. Each additional hour of cold ischemia time significantly increases the risk of graft failure and mortality following renal transplantation. Kidney Int. 2015;87:343-9.

- Maraghi E, Foroushani R, Younespour S et al. Longitudinal Assessment of Serum Creatinine Levels on Graft Survival After Renal Transplantation: Joint Modeling Approach. Nephrourol Mon 2016;8: e37666

- Sparr HJ, Vermeyen KM, Beaufort AM et al.: Early reversal of profound rocuronium-induced neuromuscular blockade by sugammadex in a randomized multicenter study efficacy, safety and pharmacokinetics. Anesthesiology, 2007; 106: 935-43.

- Tas N, Korkmaz H, Yagan O, Korkmaz M: Effect of Sugammadex on postoperative bleeding and coagulation parameters after septoplasty: A randomised prospective study. Med Sci Monit, 2015; 21: 2382-86.

- Flockton EA, Mastronardi P, Hunter JM et al.: Reversal of rocuronium-induced neuromuscular block with sugammadex is faster than reversal of cisatracurium-induced block with neostigmine. $\mathrm{Br} \mathrm{J}$ Anaesth, 2008; 100: 622-30. 


\section{Tables}

\begin{tabular}{|l|c|c|}
\hline & Roc + Sug (30 pts) & Cis + Neo (36 pts) \\
\hline Age (yr) & $52(39-59)$ & $50(41-61)$ \\
\hline Gender (M/F) & $28 / 8$ & $21 / 9$ \\
\hline BMI & $26(24-30)$ & $24(23-30)$ \\
\hline Donor's age (yr) & $49(40-56)$ & $48(41-56)$ \\
\hline Ischemic time (hr) & $10(8-14)$ & $9(7-14)$ \\
\hline Karpinski score (pts) & 4 & 3 \\
\hline Marginal transplantation & 2 & 2 \\
\hline Single transplantation & 30 & 35 \\
\hline Double transplanation & 0 & 1 \\
\hline Creatinine (mg/dl) & $7.95(5.65-11.25)$ & $7.1(5,4-9.10)$ \\
\hline Urea (mg/dl) & $26.5(14-72)$ & $60(42-79)$ \\
\hline Need of postoperative dialysis (pts) & 7 & 10 \\
\hline
\end{tabular}

Table 1: characteristics of included patients collected at the day of the kidney transplantation. Pts: patients, Roc + Sug: rocuronium + sugammadex, Cis + Neo: cisatracurium + neostigmine, yr: years, M/F: male/ female, hr: hours, pts: points. Data were reported as frequencies, median and range interquartile.

\begin{tabular}{|l|l|l|l|l|}
\hline \multicolumn{5}{|c|}{ Regression coefficients } \\
\hline & Univariate Analysis & $\mathrm{p}$ & Multivariate Analysis & $\mathrm{p}$ \\
\hline & $0.062(0.021-0.104)$ & 0.004 & $-0.117(-0.223-$ & 0.031 \\
& & & $-0.011)$ \\
\hline Creatinine 24 hr & $0.082(0.050-0.115)$ & 0.000 & & \\
\hline Creatinine 48 hr & $0.071(0.039-0.103)$ & 0.000 & & \\
\hline Creatinine 72 hr & $0.003(0.001-0.005)$ & 0.005 & $0.005(0.001-0.009)$ & 0.016 \\
\hline Urea 48 hr & $0.002(0.000-0.004)$ & 0.04 & & \\
\hline Urea 72 hr & $0.422(0.188-0.655)$ & 0.001 & & \\
\hline $\begin{array}{l}\text { Dialysis post } \\
\text { transplantation }\end{array}$ & $\begin{array}{l}-0.050(-0.086- \\
-0.013)\end{array}$ & 0.009 & & \\
\hline Na 6 hr & $-0.045(-0.08--0.011)$ & 0.011 & & \\
\hline Na 12 hr & $-0.041(-0.074-$ & 0.014 & & \\
\hline Na 24 hr & $-0.008)$ & & \\
\hline K 0 hr & $0.126(0.005-0.247)$ & 0.042 & & \\
\hline K 6 hr & $0.221(0.040-0.401)$ & 0.017 & & \\
\hline K 24 hr & $0.025(0.000-0.050)$ & 0.048 & & \\
\hline Diuresis day 1 & $0.000(0.000-0.000)$ & 0.009 & & \\
\hline Diuresis day 2 & $0.000(0.000-0.000)$ & 0.001 & & \\
\hline Diuresis day 3 & $0.000(0.000-0.000)$ & 0.001 & & \\
\hline Diuresis day 4 & $0.000(0.000-0.000)$ & 0.008 & & \\
\hline Diuresis day 5 & $0.000(0.000-0.000)$ & 0.002 & & \\
\hline Diuresis day 6 & $0.000(0.000-0.000)$ & 0.023 & & \\
\hline Diuresis day 7 & $0.000(0.000-0.000)$ & 0.014 & & \\
\hline
\end{tabular}

Table 2: univariate and multivariate analyses for the risk of post-transplantation renal injury. 
Figures

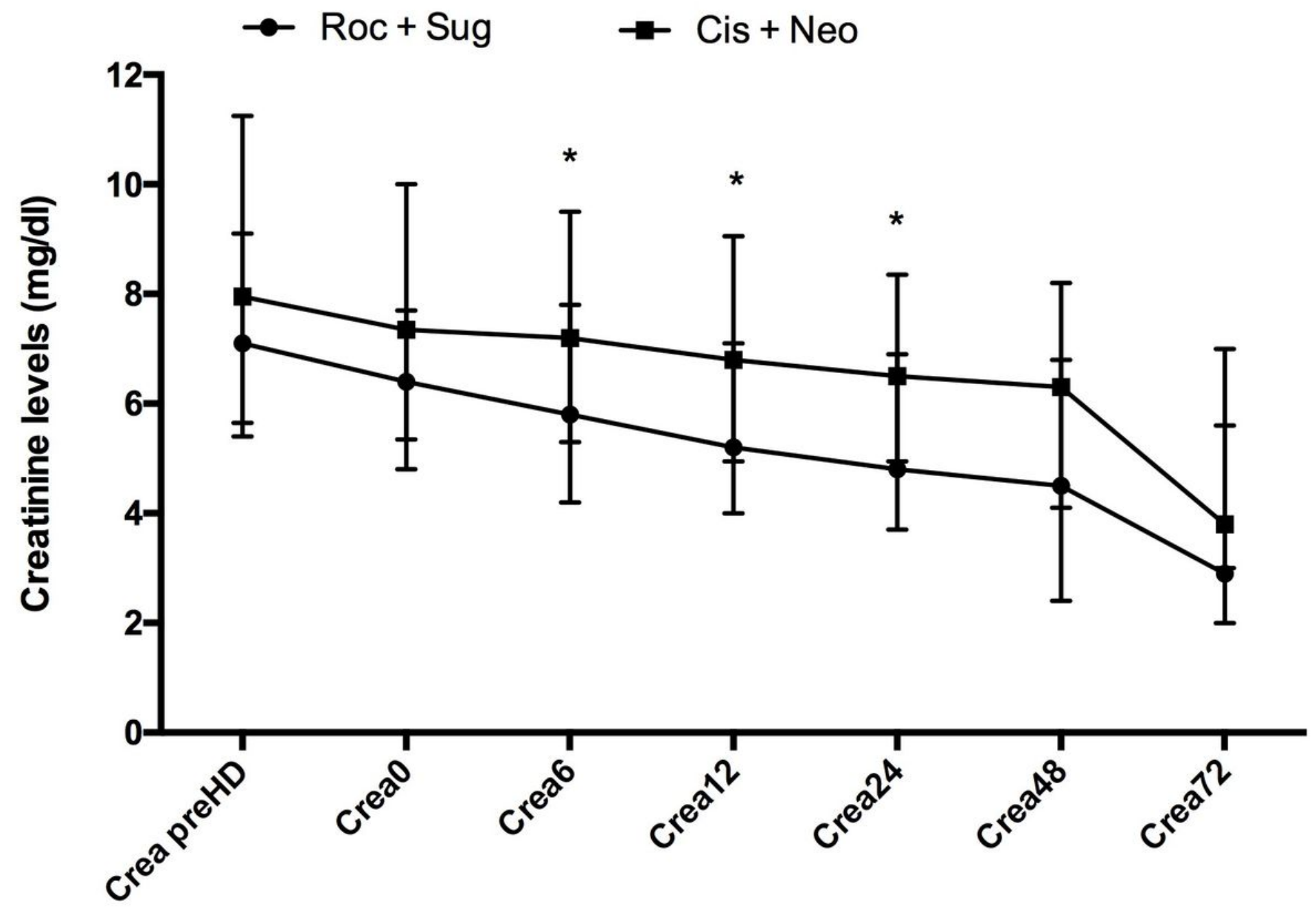

Figure 1

Blood creatinine levels before and after the kidney transplantation. Crea: creatinine, roc + sug: rocuronium + sugammadex, cis + neo: cisatracurium + neostigmine. ${ }^{*} p<0.05$. 


\section{$\rightarrow \mathrm{Cis}+\mathrm{NeO} \rightarrow \mathrm{Roc}+\mathrm{Sug}$}

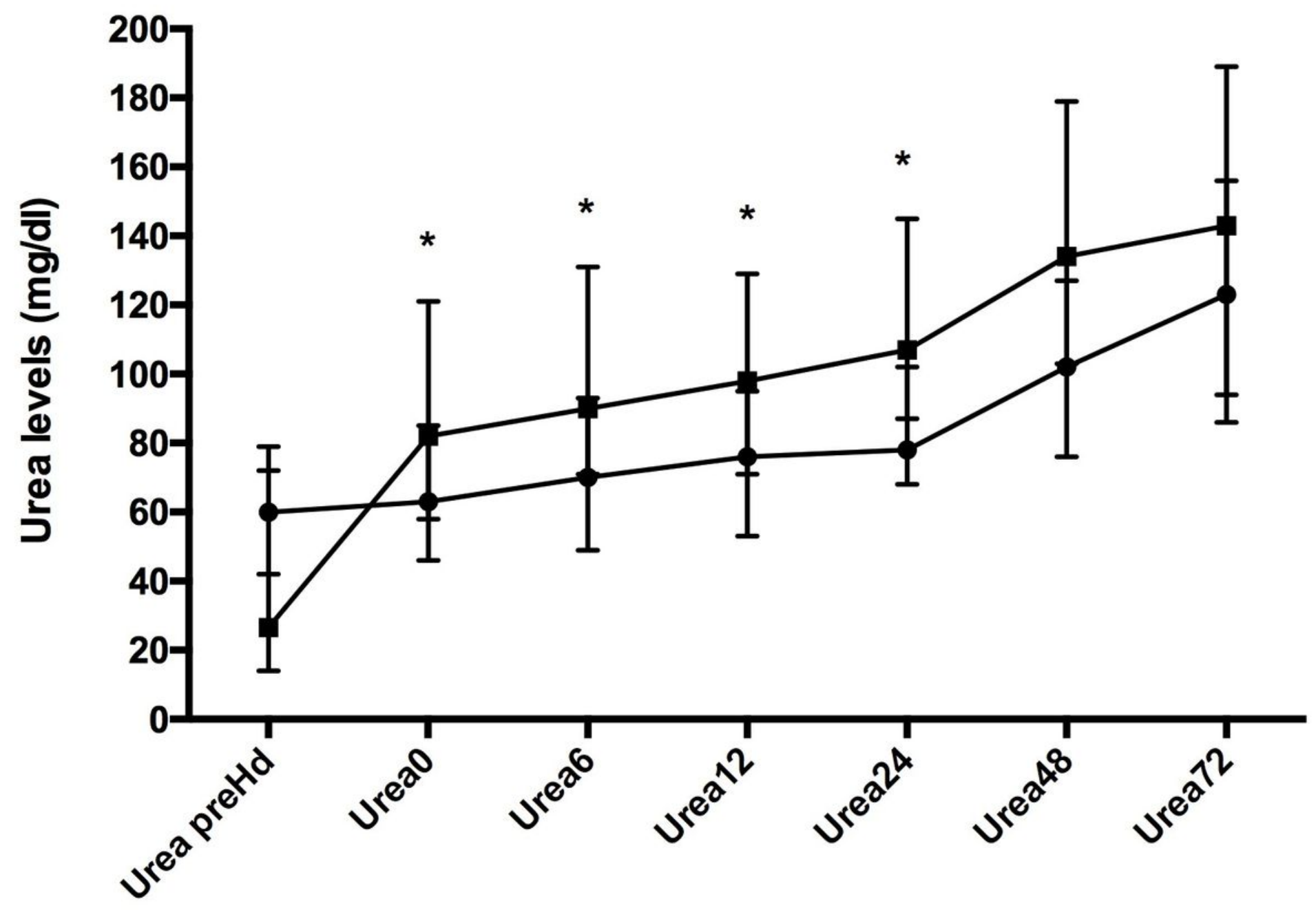

Figure 2

Blood urea levels before and after the kidney transplantation. Crea: creatinine, roc + sug: rocuronium + sugammadex, cis + neo: cisatracurium + neostigmine. ${ }^{*} p<0.05$. 


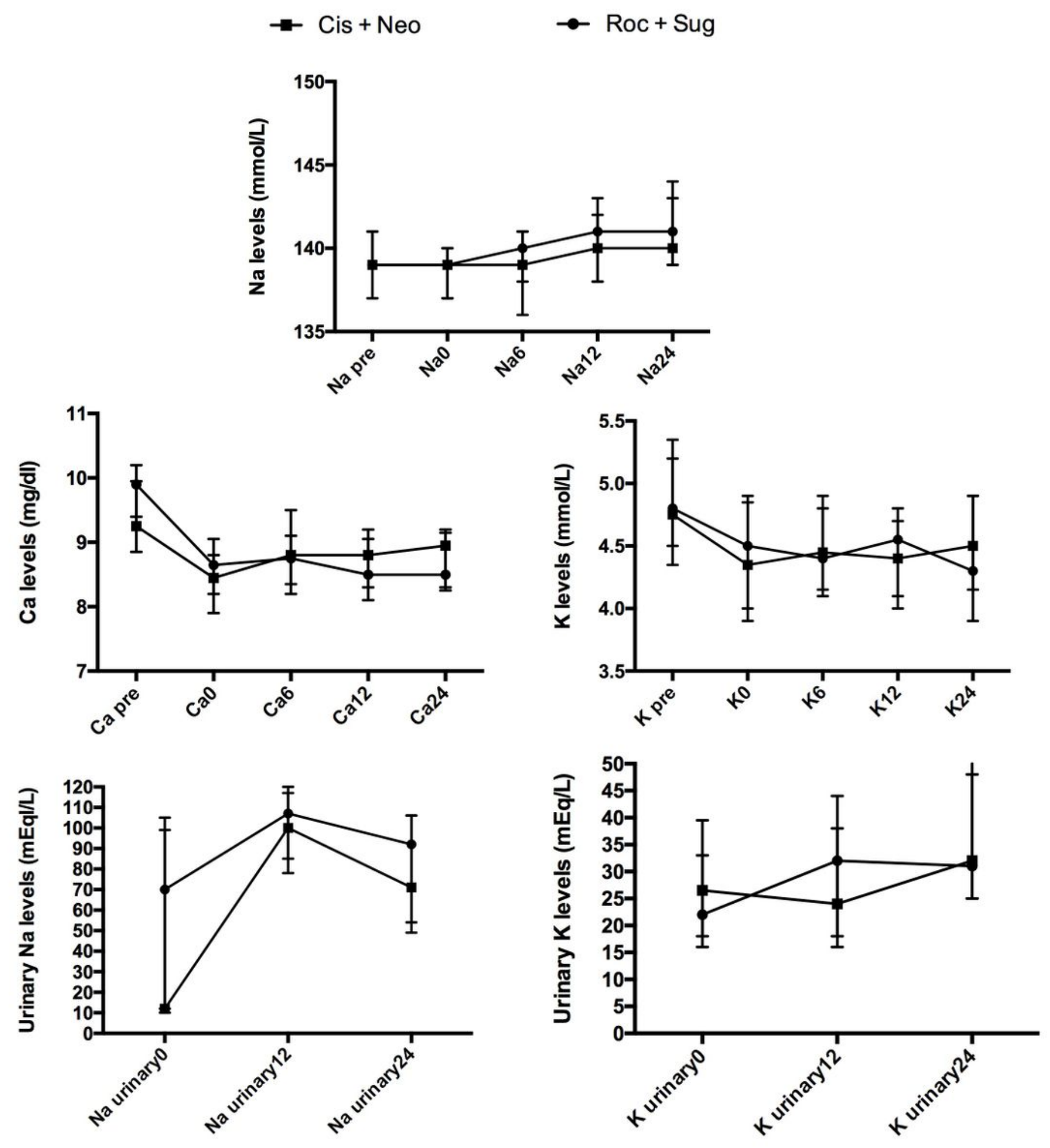

Figure 3

Blood sodium, blood potassium, blood calcium levels and urinary sodium and urinary potassium levels before and after the kidney transplantation. Crea: creatinine, roc + sug: rocuronium + sugammadex, cis + neo: cisatracurium + neostigmine. 


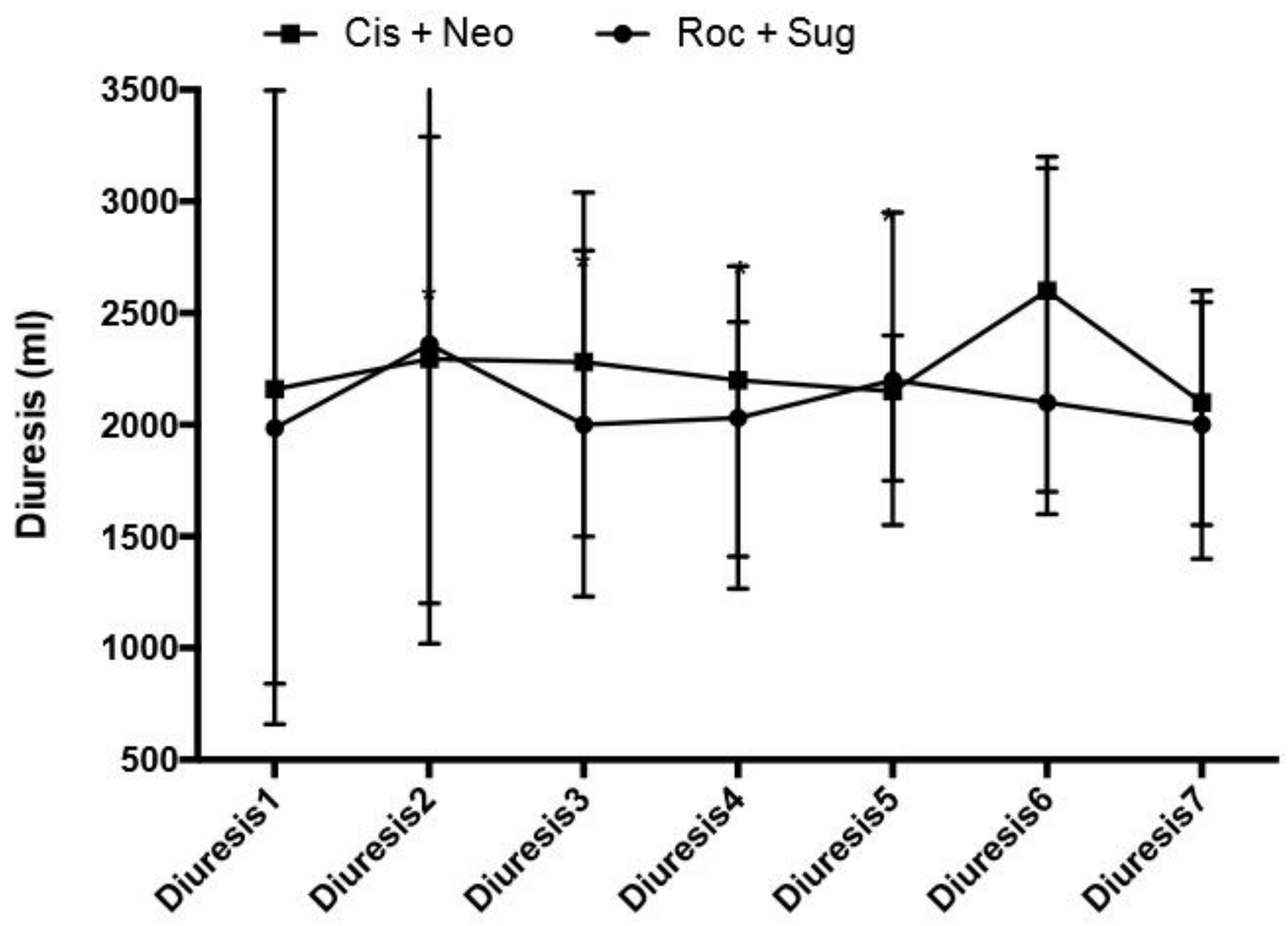

Figure 4

Diuresis levels before and after the kidney transplantation. Crea: creatinine, roc + sug: rocuronium + sugammadex, cis + neo: cisatracurium + neostigmine. 